\title{
Risk factors for symptomatic urinary tract infections in individuals with chronic neurogenic lower urinary tract dysfunction
}

\author{
$\mathrm{J} \mathrm{Krebs}^{1}, \mathrm{~J}$ Wöllner${ }^{2}$ and J Pannek ${ }^{2}$
}

Study design: Retrospective investigation.

Objectives: To investigate the association of patient and injury characteristics, as well as bladder management, with the occurrence of patient-reported, symptomatic urinary tract infection(s) UTI(s) in patients with chronic neurogenic lower urinary tract dysfunction (NLUTD).

Setting: Tertiary urologic referral center.

Methods: The patient database was screened for patients with chronic (>12 months) NLUTD who had presented between 2008 and 2012. Patient characteristics, bladder evacuation management, the annual number of patient-reported, symptomatic UTIs and the type of prophylactic treatment to prevent UTIs were collected. Binary logistic regression analysis was used to investigate the effects of the investigated risk factors on the occurrence of symptomatic UTI(s) and recurrent symptomatic UTIs ( $\geqslant 3$ annual UTIs).

Results: The data of 1104 patients with a mean NLTUD duration of $20.3 \pm 11.6$ years were investigated. The evacuation method was a significant $(P \leqslant 0.004)$ predictor for the occurrence of symptomatic UTI and recurrent symptomatic UTIs. The greatest annual number of symptomatic UTIs was observed in patients using transurethral indwelling catheters, and the odds of experiencing a UTI and recurrent UTIs were increased more than 10- and 4-fold, respectively. The odds of a UTI or recurrent UTIs were also increased significantly $(P \leqslant 0.014)$ in patients using intermittent catheterization $(I C)$. Botulinum toxin injections into the detrusor increased the odds of a UTI 10-fold ( $P=0.03$ ).

Conclusions: The bladder evacuation method is the main predictor for symptomatic UTIs in individuals with NLUTD. Transurethral catheters showed the highest odds of symptomatic UTI and should be avoided whenever possible.

Spinal Cord (2016) 54, 682-686; doi:10.1038/sc.2015.214; published online 1 December 2015

\section{INTRODUCTION}

Urinary tract infections (UTIs) remain one of the most common morbidities in individuals with neurogenic lower urinary tract dysfunction (NLUTD) $\cdot^{1-4}$ Approximately one in five individuals with NLTUD suffers from recurrent UTIs, which affects the quality of life considerably. ${ }^{5,6}$ The increased risk of UTIs in individuals with NLUTD is the result of the impaired storage and voiding functions. Thus, the key to controlling UTIs in individuals with NLUTD is to optimize their bladder management and to eliminate sources of infection, such as bladder stones or foreign bodies for example., ${ }^{7,8}$ Furthermore, the administration of preventive or protective measures can be optimized by identifying individuals at risk and anticipating times of increased risk.

Many different risk factors for UTIs such as patient or injury characteristics, psychosocial or behavioral aspects, bladder evacuation method or residual urine have been considered. ${ }^{9-12}$ However, there is insufficient evidence to assess the relevance of most of these factors. ${ }^{9}$ Only injury severity and indwelling catheterization have been demonstrated to be associated with UTIs. ${ }^{10,13,14}$

We have therefore investigated the association of patient and injury characteristics, as well as bladder management, with the occurrence of patient-reported, symptomatic UTIs in patients with chronic NLUTD. The following hypotheses were tested: gender, age, injury severity, injury duration, injury etiology, bladder evacuation method and botulinum toxin (BTx) injections into the detrusor have a significant effect on the occurrence of (1) symptomatic UTI(s) and (2) recurrent symptomatic UTIs ( $\geqslant 3$ annual UTIs).

\section{SUBJECTS AND METHODS}

\section{Subjects and collected data}

The patient database of a tertiary urologic referral center was screened for patients with chronic ( $>12$ months) NLUTD resulting from traumatic or nontraumatic spinal cord injury, myelomeningocele or multiple sclerosis, who had presented for a routine urodynamic investigation between April 2008 and March 2012. During the visit, data concerning bladder evacuation details, medication and symptomatic UTI were routinely collected using a standardized form. The patients were asked to report the number of symptomatic UTIs they had experienced during the past 12 months. Self-reported symptomatic UTIs were considered clinically relevant, because patients present for UTI treatment when they experience symptoms. A UTI was defined as a combination of clinical symptoms and laboratory findings (leukocyturia and bacteriuria). ${ }^{6}$ The following signs and symptoms were considered signs and symptoms of a UTI: fever without another cause, new onset of incontinence or voiding problems,

${ }^{1}$ Clinical Trial Unit, Swiss Paraplegic Centre, Nottwil, Switzerland and ${ }^{2}$ Department of Neurourology, Swiss Paraplegic Centre, Nottwil, Switzerland

Correspondence: Professor J Pannek, Department of Neurourology, Swiss Paraplegic Centre, Schweizer Paraplegiker Zentrum, Guido A. Zäch Strase 1, Nottwil CH-6207, Switzerland.

E-mail: juergen.pannek@paraplegie.ch

Received 27 July 2015; revised 12 October 2015; accepted 4 November 2015; published online 1 December 2015 
decreased bladder capacity, increased voiding or catheterization frequency, kidney or bladder discomfort, cloudy urine or hematuria without another cause. $^{15}$

Patient characteristics, bladder evacuation methods, the annual number of symptomatic UTIs, the type of prophylactic treatment to prevent UTIs and the type of detrusor relaxation treatment were collected from paper and electronic patient charts. The annual number of symptomatic UTIs was collected for 3 consecutive years, to attenuate the effect of a single observation. Thus, patients with a follow-up shorter than 3 years were excluded.

The study had been approved by the local ethics committee, and all applicable institutional and governmental regulations concerning the ethical use of the data were followed.

\section{Statistical analyses}

The investigated patients were grouped for age and injury duration using 15- and 5-year increments, respectively. ${ }^{16}$ According to international recommendations, ${ }^{16}$ injury severity was categorized using the following groups: (1) American Spinal Injury Association Impairment Scale (AIS) D, (2) AIS A-C paraplegia, (3) AIS A-C low-level tetraplegia (C8-C5) and (4) AIS A-C highlevel tetraplegia $(\mathrm{C} 4-\mathrm{C} 1)$. The average annual number of symptomatic UTIs was determined by calculating the median of the values from 3 years, to attenuate the effect of a single observation. Binary logistic regression analysis was used to investigate the effects of gender, age, injury severity, duration and etiology, bladder evacuation method, detrusor relaxation by BTx injections and UTI prophylaxis on the occurrence of symptomatic UTI(s) ( 0 or $>0$ annual UTI) and recurrent symptomatic UTIs ( $<3$ or $\geqslant 3$ annual UTIs). The occurrence rate of symptomatic UTI(s) and recurrent symptomatic UTIs and the $95 \%$ confidence intervals (CIs) were calculated for the significant predictors using the Clopper-Pearson exact binomial method. Normal distribution was assumed based on the central limit theorem, and data are thus presented as mean with standard deviation and 95\% CI where appropriate. The statistical analyses were performed using the SPSS software (version 18.0.3; IBM, Somers, NY, USA). A $P$-value of $\leqslant 0.05$ was considered significant.

\section{RESULTS}

We identified 1324 patients with chronic NLUTD who had presented at least once at our center between April 2008 and March 2012 for a routine urodynamic investigation. A total of 219 patients were excluded because their follow-up was shorter than 3 years. One patient was excluded because no data was available concerning the annual number of UTIs. Thus, the data of 1104 patients with a mean age of $48 \pm 14$ years (range $12-87$ years) were investigated. The mean duration of NLTUD was 20.3 \pm 11.6 years (range 4.0-63.4 years). The characteristics of the evaluated patients are presented in Table 1.

The bladder evacuation method, BTx injections into the detrusor and prophylactic treatment to prevent UTI(s) were significant $(P \leqslant 0.049)$ predictors for the occurrence of symptomatic UTI(s) and recurrent symptomatic UTIs (Table 2). The odds of experiencing a symptomatic UTI in patients voiding via transurethral indwelling catheters (TUC) $(18 / 1104,1.6 \%)$ were increased more than 10-fold compared with patients voiding spontaneously (71/1104, 6\%) (Table 2). The occurrence rate of a symptomatic UTI was $83.3 \%$ and $29.6 \%$ for TUC and spontaneous voiding, respectively (Table 3 ). In patients using IC $(427 / 1104,39 \%)$, suprapubic catheterization (SPC) $(120 / 1104,11 \%)$ or triggered reflex voiding (299/1104, 27\%), the odds of a symptomatic UTI were also increased significantly (Table 2). Approximately $70 \%$ of patients using IC suffered at least one symptomatic UTI per year (Table 3). Bladder voiding by TUC increased the odds of recurrent symptomatic UTIs more than fivefold compared with spontaneous voiding (Table 2). Half of the patients using TUC and $\sim 30 \%$ of the patients using IC experienced more than two symptomatic UTIs per year (Table 3).
BTx injections increased the odds of a symptomatic UTI and recurrent symptomatic UTIs 1.7 and 1.55 times (Table 2). The occurrence rate of a symptomatic UTI in patients receiving BTx injections was $76.8 \%$ compared with $59.0 \%$ in those who did not (Table 3).

Table 1 The characteristics of the evaluated patients

\begin{tabular}{llll}
\hline Variables & Groups & N & $\%$ \\
\hline \multirow{2}{*}{ Gender } & Women & 283 & 25.6 \\
& Men & 821 & 74.
\end{tabular}

Age (years)

$\begin{array}{ll}11-30^{\mathrm{a}} & 129 \\ 31-45 & 327 \\ 46-60 & 4 \\ 61-75 & 203 \\ >75 & \end{array}$

$129 \quad 11.7$

$327 \quad 29.6$

$413 \quad 37.4$

$203 \quad 18.4$

32

2.9

Injury severity

C1-C4 AIS A-C $73 \quad 6.6$

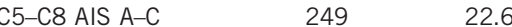

T1-S5 AIS A-C $\quad 622 \quad 56.3$

AIS Da

$87 \quad 7.9$

Not determinable

73

6.6

Injury duration (years)

$<6$

$6-10$

46

4.2

$11-15$

194

17.6

$16-20$

$189 \quad 17.1$

21-25 $143 \quad 13.0$

26-30 $126 \quad 11.4$

$\begin{array}{lll}31-35 & 87 & 7.9\end{array}$

$\begin{array}{lll}36-40 & 59 & 5.3\end{array}$

$41-45$

$>45$

41

37

3.7

3.4

Etiology

Traumatic SCla

Non-traumatic SCl

$889 \quad 80.5$

MMC

MS

6.3

7.8

5.4

Evacuation method

Spontaneous $^{\mathrm{a}}$

SARS

Straining

Reflex

$I^{b}$

SPC

TUC

69

86

60

6.4

8.4

6.9

27.1

38.7

10.9

1.6

Prophylaxis

No

64.2

Prophylaxis

Yes

709

35.8

Botulinum toxin

No

959

86.9

138

12.5

Not determinable

Abbreviations: AIS, American Spinal Cord Injury Association Impairment Scale; botulinum toxin, botulinum toxin injections into detrusor; $\mathrm{C} 1-\mathrm{C} 4$, first to fourth cervical spinal cord segment; C5-C8, cervical spinal cord segments 5 to 8 ; IC, intermittent catheterization;

MMC, myelomeningocele; MS, multiple sclerosis; prophylaxis, prophylactic treatment to prevent UTIs; reflex, triggered reflex voiding; SARS, sacral anterior root stimulation; $\mathrm{SCl}$, spinal cord injury; SPC, suprapubic catheterization; straining, voiding by abdominal straining (Valsalva); T1-S5, first thoracic to fifth sacral spinal cord segment; TUC, transurethral indwelling catheter. aReference category for binary logistic regression.

bSingle use, hydrophilic catheters in 423/427 patients (99\%). 
Table 2 Evaluating the effect of different predictors on the occurrence of urinary tract infections using binary logistic regression

\begin{tabular}{|c|c|c|c|c|c|c|}
\hline \multirow[t]{2}{*}{ Predictors } & \multirow[t]{2}{*}{ P-value } & \multicolumn{2}{|c|}{ UTI(s) } & \multirow[t]{2}{*}{ P-value } & \multicolumn{2}{|c|}{ Recurrent UTIs } \\
\hline & & $\operatorname{Exp}(\mathrm{B})$ & $95 \% \mathrm{Cl} \operatorname{Exp}(\mathrm{B})$ & & $\operatorname{Exp}(\mathrm{B})$ & $95 \% \mathrm{Cl} \operatorname{Exp}(\mathrm{B}$ \\
\hline Gender (men/women) & 0.3 & 1.20 & $0.85-1.68$ & 0.7 & 1.08 & $0.74-1.58$ \\
\hline Age $^{a}$ & 0.11 & & 0.09 & & & \\
\hline Injury severitya & 0.5 & & 0.89 & & & \\
\hline Injury duration ${ }^{a}$ & 0.16 & & 0.07 & & & \\
\hline Etiology ${ }^{a}$ & 0.1 & & 0.21 & & & \\
\hline Evacuation method ${ }^{a}$ & $<0.001$ & & 0.01 & & & \\
\hline Spontaneous & \multicolumn{3}{|c|}{ Reference category } & \multicolumn{3}{|c|}{ Reference category } \\
\hline TUC & 0.002 & 10.27 & $2.34-45.12$ & 0.018 & 5.20 & $1.33-20.36$ \\
\hline SPC & 0.018 & 2.44 & $1.16-5.14$ & 0.46 & 1.47 & $0.53-4.08$ \\
\hline IC & $<0.001$ & 3.54 & $1.79-7.00$ & 0.04 & 2.66 & $1.05-6.76$ \\
\hline Reflex & 0.013 & 2.56 & $1.21-4.79$ & 0.13 & 2.10 & $0.81-5.40$ \\
\hline Straining & 0.22 & 1.66 & $0.74-3.68$ & 0.66 & 1.28 & $0.42-3.91$ \\
\hline SARS & 0.15 & 1.77 & $0.82-3.82$ & 0.88 & 1.09 & $0.37-3.19$ \\
\hline BTx (no/yes) & 0.026 & 1.70 & $1.07-2.71$ & 0.049 & 1.55 & $1.00-2.40$ \\
\hline Prophylaxis & $<0.001$ & 1.96 & $1.46-2.62$ & 0.001 & 1.70 & $1.25-2.30$ \\
\hline
\end{tabular}

Abbreviations: AIS, American Spinal Cord Injury Association Impairment Scale; BTx, botulinum toxin detrusor injections; Cl, confidence interval; C1-C4, cervical spinal cord segments 1 to 4; C5-C8, cervical spinal cord segments 5 to 8; Exp $(B)$, odds ratio predicted by model; IC, intermittent catheterization; MMC, myelomeningocele; MS, multiple sclerosis; prophylaxis, prophylactic treatment to prevent urinary tract infections; recurrent UTIs, $\geqslant 3$ annual urinary tract infections; reflex, triggered reflex voiding; SARS, sacral anterior root stimulation; SCI, spinal cord injury;

SPC, suprapubic catheterization; straining, voiding by abdominal straining (Valsalva); T1-S5, thoracic spinal cord segment 1 to sacral segment 5; TUC, transurethral indwelling catheter;

$\mathrm{UTI}(\mathrm{s}),>0$ annual urinary tract infection(s).

$P$-values $\leqslant 0.05$ are indicated in bold

${ }^{a}$ General odds are not calculated.

Table 3 Occurrence of urinary tract infections for the different categories of the significant predictors

\begin{tabular}{|c|c|c|c|c|c|}
\hline \multirow[t]{2}{*}{ Significant predictors } & \multicolumn{2}{|c|}{ UTI } & \multicolumn{2}{|c|}{ Recurrent UTIs } & \multirow{2}{*}{$\begin{array}{c}\text { Total } \\
\% / n\end{array}$} \\
\hline & $\% / 95 \% \mathrm{Cl}$ & $n$ & $\% / 95 \% \mathrm{Cl}$ & $n$ & \\
\hline \multicolumn{6}{|l|}{ Evacuation method } \\
\hline Spontaneous & 29.6/19.3-41.6 & 21 & 9.9/4.1-19.3 & 7 & 100/71 \\
\hline Straining & $47.4 / 22.0-39.0$ & 36 & $14.5 / 4.7-15.8$ & 11 & $100 / 120$ \\
\hline SARS & $53.8 / 43.1-64.2$ & 50 & $15.1 / 8.5-24.0$ & 14 & 100/93 \\
\hline SPC & 58.3/49.0-67.3 & 70 & 17.5/11.2-25.5 & 21 & $100 / 120$ \\
\hline Reflex & $61.5 / 55.8-67.1$ & 184 & 25.8/20.9-31.1 & 77 & $100 / 299$ \\
\hline IC & 70.5/65.9-74.8 & 301 & $31.2 / 26.8-35.8$ & 133 & $100 / 427$ \\
\hline TUC & 83.3/58.6-96.4 & 15 & $50.0 / 26.0-74.0$ & 9 & $100 / 18$ \\
\hline Total & $61.3 / 58.4-64.2$ & 677 & $24.6 / 22.1-27.3$ & 272 & $100 / 1104$ \\
\hline \multicolumn{6}{|l|}{ Botulinum toxin } \\
\hline No & 59.0/55.8-62.1 & 566 & 23.0/20.4-25.8 & 221 & $100 / 959$ \\
\hline Yes & 76.8/68.9-83.6 & 106 & $36.2 / 28.2-44.8$ & 50 & $100 / 138$ \\
\hline Not determinable & 71.4/29.0-96.3 & 5 & 14.3/0.4-57.9 & 1 & $100 / 7$ \\
\hline \multicolumn{6}{|l|}{ Prophylaxis } \\
\hline No & $55.3 / 51.5-59.0$ & 392 & 20.5/17.5-23.6 & 145 & $100 / 709$ \\
\hline Yes & $72.2 / 67.4-76.5$ & 285 & $32.2 / 27.6-37.0$ & 127 & $100 / 395$ \\
\hline
\end{tabular}

Abbreviations: Botulinum toxin, botulinum toxin injections into the detrusor; $\mathrm{Cl}$, confidence interval; IC, intermittent catheterization; prophylaxis, prophylactic treatment to prevent urinary tract infections; recurrent UTIs, $\geqslant 3$ annual UTIs; reflex, triggered reflex voiding; SARS, sacral anterior root stimulation; SPC, suprapubic catheterization; training, voiding by abdominal straining (Valsalva); TUC, transurethral indwelling catheter; UTI, >0 annual UTI.

A total of 395 patients $(35.8 \%)$ were receiving prophylactic treatment for the prevention of UTIs (Table 1). The different types of prophylactic treatment are presented in Table 4. Phytotherapy and urine acidification were the two most common prophylactic regimens. The percentage of patients taking antibiotics for infection prophylaxis was $11 \%$ (Table 4 ). The odds of a symptomatic UTI and recurrent symptomatic UTIs were increased $~ 2$-fold and 1.7 times, respectively, with prophylactic treatment (Table 2).

\section{DISCUSSION}

We have investigated the effects of patient and injury characteristics, as well as bladder management, on the occurrence of patient-reported, 
Table 4 Prophylactic treatment for the prevention of urinary tract infections used by evaluated patients

\begin{tabular}{|c|c|c|c|}
\hline Category & Agent & $\mathrm{N}$ & $\%$ \\
\hline \multirow{5}{*}{ Phytotherapy } & Cranberries & 215 & 54.3 \\
\hline & Horseradish and nasturtium & 10 & 2.5 \\
\hline & Bearberry & 2 & 0.5 \\
\hline & Undetermined & 1 & 0.25 \\
\hline & Total & 228 & 57.6 \\
\hline \multirow[t]{3}{*}{ Urine acidification } & Methionine & 177 & 44.7 \\
\hline & Cider vinegar & 2 & 0.5 \\
\hline & Total & 179 & 45.2 \\
\hline \multirow[t]{11}{*}{ Antibiotics } & Nitrofurantoin & 19 & 4.8 \\
\hline & Fuoroquinolone & 9 & 2.3 \\
\hline & Trimethoprim, sulfamethoxazole & 6 & 1.5 \\
\hline & $\begin{array}{l}\text { Trimethoprim, sulfamethoxazole } \\
\text { /fluoroquinolone }\end{array}$ & 2 & 0.5 \\
\hline & Trimethoprim, sulfamethoxazole/fosfomycin & 2 & 0.5 \\
\hline & Fosfomycin/cephalosporin & 2 & 0.5 \\
\hline & Trimethoprim, sulfamethoxazole/nitrofurantoin & 1 & 0.25 \\
\hline & Trimethoprim, sulfamethoxazole/rifampicin & 1 & 0.25 \\
\hline & Cephalosporin & 1 & 0.25 \\
\hline & Rifampicin & 1 & 0.25 \\
\hline & Total & 44 & 11.1 \\
\hline \multirow[t]{3}{*}{ Vaccination } & Escherichia coli & 14 & 3.5 \\
\hline & E. coli, miscellaneous & 1 & 0.25 \\
\hline & Total & 15 & 3.8 \\
\hline Hhomeopathy & NA & 4 & 1.0 \\
\hline \multirow[t]{3}{*}{ Antibacterial } & Methenamine hippurate & 2 & 0.5 \\
\hline & D-mannose & 2 & 0.5 \\
\hline & Total & 4 & 1.0 \\
\hline Bladder irrigation & Sodium chloride solution & 2 & 0.5 \\
\hline Undetermined & NA & 1 & 0.25 \\
\hline
\end{tabular}

Abbreviations: Miscellaneous, Morganella morganii, Proteus mirabilis, Klebsiella pneumoniae, Enterococcus faecalis; NA, not available.

The sum of the percentages exceeds $100 \%$ because some patients are using several different prophylactic treatments.

symptomatic UTI in 1104 patients with chronic NLUTD (mean duration 20 years). The odds of experiencing a symptomatic UTI ( $>0$ annual UTI) or recurrent symptomatic UTIs ( $\geqslant 3$ annual UTIs) were significantly increased in patients using TUC or IC compared with those voiding spontaneously. Furthermore, BTx injections into the detrusor increased the odds of a symptomatic UTI or recurrent symptomatic UTIs significantly.

The bladder evacuation method was identified as the main predictor for the occurrence of symptomatic UTI(s). In patients using TUC, the odds of a symptomatic UTI and recurrent symptomatic UTIs were increased more than 10- and 5-fold, respectively. Accordingly, the highest percentage of patients affected with symptomatic UTI(s) $(83 \%)$ was observed in the TUC group. This is in accordance with other studies. ${ }^{9}, 10,14$ Esclarin de Ruz et al. ${ }^{10}$ have reported an odds ratio of $\sim 8$ for UTIs in patients with acute NLUTD using TUC. Thus, present and previous results confirm the increased risk of UTIs for TUC. Furthermore, high UTI rates have also been reported for voiding by SPC (i.e. 73\%). ${ }^{13,14}$ In the present study, the odds of a symptomatic UTI were increased 2.4 times in patients using SPC, and 58\% were affected by at least one symptomatic UTI. However, there is also a report of reduced odds of UTI for SPC. ${ }^{10}$ Several reports have documented lower UTI rates in patients using IC compared with those using TUC. ${ }^{9,10,13,14}$ However, the UTI rates for IC (35\%) were increased compared with spontaneous voiding. ${ }^{10,13}$ In the present study, both the odds of a symptomatic UTI and recurrent symptomatic UTIs were increased in the IC group, and $71 \%$ were affected by UTIs. Furthermore, both the rates of symptomatic UTI and the odds for symptomatic UTI(s) were higher for IC compared with SPC, which is in accordance with a previous report. ${ }^{17}$ We have also observed a considerable rate of symptomatic UTI in patients using triggered reflex voiding (i.e. 62\%). Similarly, other investigators have reported increased UTI rates for triggered reflex voiding compared with spontaneous voiding; however, the observed rates were slightly lower (i.e. 44\%). ${ }^{10,13}$ The discrepancies between previously reported and present data are the result of differences in the duration of NLUTD, definition of a UTI, calculation of the UTI rate and study design.

The occurrence rate $(77 \%)$ of symptomatic UTI(s) in patients receiving $\mathrm{BTx}$ injections into the detrusor was significantly greater compared with the rate $(59 \%)$ in those who did not. Furthermore, the odds of a symptomatic UTI and recurrent symptomatic UTIs were increased $\sim 1.5$ times, respectively. In previous investigations, a decrease in the number of patients affected by UTIs and the incidence of UTIs was observed after BTx injections. ${ }^{18,19}$ These results are not necessarily contradictory to the present ones, as patients may experience a decrease in the UTI rate after BTx injections but still suffer from UTIs more often than those who do not receive injections. Present data represent the situation under chronic BTx treatment, and thus no comparison can be made between the UTI rate before and the rate after starting BTx treatment. Furthermore, the causality between BTx injections and symptomatic UTIs should be considered cautiously because of the cross-sectional study design.

In the present study, patient and injury characteristics had no significant effect on the occurrence of symptomatic UTIs. This is largely in accordance with other reports. ${ }^{10,11,13}$ Some investigators have observed increased UTI rates in patients with motor complete spinal cord injury, ${ }^{13}$ cervical injury ${ }^{10}$ or increased functional independence. ${ }^{10}$ However, injury severity and level have a major impact on both functional independence and the chosen bladder evacuation method, and thus may represent confounding factors. We have included these factors in our statistical model.

Approximately every third patient was receiving prophylactic treatment for the prevention of UTIs. In these patients, the odds of a symptomatic UTI and recurrent symptomatic UTIs were significantly increased compared with those who did not receive prophylactic treatment. However, the occurrence of symptomatic UTIs was the reason for the prophylactic treatment and not vice versa. Yet, the prophylactic measures do not seem to be very successful in reducing the occurrence of symptomatic UTIs. Cranberry products and urine acidification using methionine were the two most common prophylactic regimens, which are reported to be the current clinical practice in the prevention of UTI in German-speaking spinal cord injury centers. ${ }^{20}$ However, based on current evidence, cranberry products or urine acidification do not reduce the occurrence of symptomatic UTIs. ${ }^{21,22}$ Long-term antibiotic treatment for UTI prevention is administered in most spinal cord injury centers. ${ }^{20}$ In the present study, $11 \%$ of the patients were taking antibiotics for UTI prevention. However, antibiotic treatment for UTI prevention is not supported by current evidence. $^{23,24}$ Moreover, the risk of antibiotic resistance is increased under long-term treatment. ${ }^{23,24}$ 
The retrospective data collection and the mediocre accuracy (i.e. $66 \%$ ) of the patient self-prediction of $\mathrm{UTI}^{25}$ contribute to the uncertainty about the true number of annual symptomatic UTIs. However, the analyses of the present study are not based on the number of annual symptomatic UTIs but on the two categories 'UTI' ( $>0$ annual UTI) and 'recurrent UTIs' ( $\geqslant 3$ annual UTIs), which can be determined accurately and reliably. The causality between predictors and symptomatic UTIs should be considered cautiously because of the cross-sectional study design. Furthermore, some possible risk factors for symptomatic UTIs, such as the urodynamic situation, residual urine or bladder stones, were not included in our analysis. However, the urodynamic situation and the presence of bladder stones or relevant residual urine volumes are examined in our patients during routine follow-up visits, and appropriate measures are initiated, if required. Finally, the reported odds ratios should be interpreted with caution because of the wide CI.

Symptomatic UTIs have a considerable effect on the quality of life of individuals with NLUTD, and identifying individuals at risk and anticipating times of increased risk are key factors in the prevention and management of UTIs. On the basis of the present results, patient and injury characteristics do not represent risk factors for the occurrence of symptomatic UTIs in individuals with NLUTD. However, the bladder evacuation method is the main predictor for the occurrence of symptomatic UTIs. We have identified long-term TUC as the primary predictor of symptomatic UTIs in individuals with NLUTD. Thus, long-term TUC should be avoided whenever possible, inasmuch as further complications (e.g. bladder stones, cancer) are associated with this evacuation method. ${ }^{26-28}$ Evacuation by SPC represents a valuable alternative, particularly with regard to symptomatic UTIs. In the present study, SPC has shown the lowest odds of symptomatic UTIs among catheter-based evacuation methods. The gold standard for bladder evacuation in individuals with NLUTD and sufficient manual dexterity is IC. ${ }^{29}$ However, present results indicate that the odds of symptomatic UTIs are increased considerably for IC. Even though this does not represent a reason to abandon IC, protective and preventive measures should be initiated timely in these patients. The same conclusion can be drawn for triggered reflex voiding, which was associated with increased odds of a symptomatic UTI. Thus, further research regarding prophylactic measures to prevent symptomatic UTIs should be initiated, as currently used measures do not seem to be effective. Finally, increased odds of a symptomatic UTI and recurrent symptomatic UTIs should be anticipated in patients receiving BTx injections into the detrusor and appropriate prophylactic measures initiated.

\section{CONCLUSIONS}

The bladder evacuation method, rather than patient or injury characteristics, is the main predictor for the occurrence of symptomatic UTIs in individuals with NLUTD. Long-term TUC showed the highest odds of symptomatic UTI(s) and should thus be avoided whenever possible.

\section{DATA ARCHIVING}

There were no data to deposit.

\section{CONFLICT OF INTEREST}

The authors declare no conflict of interest.
1 Nicolle LE. Urinary tract infections in patients with spinal injuries. Curr Infect Dis Rep 2014; 16: 390.

2 Jahromi MS, Mure A, Gomez CS. UTIs in patients with neurogenic bladder. Curr Urol Rep 2014; 15: 433

3 Whiteneck GG, Charlifue SW, Frankel HL, Fraser MH, Gardner BP, Gerhart KA et al. Mortality, morbidity, and psychosocial outcomes of persons spinal cord injured more than 20 years ago. Paraplegia 1992; 30: 617-630.

4 Wyndaele JJ, Brauner A, Geerlings SE, Bela K, Peter T, Bjerklund-Johanson TE. Clean intermittent catheterization and urinary tract infection: review and guide for future research. BJU Int 2012; 110: E910-E917.

5 Biering-Sorensen F, Nielans HM, Dorflinger T, Sorensen B. Urological situation five years after spinal cord injury. Scand J Urol Nephrol 1999; 33: 157-161.

6 Goetz LL, Cardenas DD, Kennelly M, Bonne Lee BS, Linsenmeyer T, Moser C et al. International Spinal Cord Injury Urinary Tract Infection Basic Data Set. Spinal Cord 2013; 51: 700-704.

7 Goetz LL, Klausner AP. Strategies for prevention of urinary tract infections in neurogenic bladder dysfunction. Phys Med Rehabil Clin N Am 2014; 25: 605-618.

8 Biering-Sorensen F, Bagi P, Hoiby N. Urinary tract infections in patients with spinal cord lesions: treatment and prevention. Drugs 2001; 61: 1275-1287.

9 Shekelle PG, Morton SC, Clark KA, Pathak M, Vickrey BG. Systematic review of risk factors for urinary tract infection in adults with spinal cord dysfunction. J Spinal Cord Med 1999; 22: 258-272.

10 Esclarin De Ruz A, Garcia Leoni E, Herruzo Cabrera R. Epidemiology and risk factors for urinary tract infection in patients with spinal cord injury. J Urol 2000; 164: 1285-1289.

11 Waites KB, Canupp KC, DeVivo MJ. Epidemiology and risk factors for urinary tract infection following spinal cord injury. Arch Phys Med Rehabil 1993; 74: 691-695.

12 Krebs J, Bartel P, Pannek J. Residual urine volumes after intermittent catheterization in men with spinal cord injury. Spinal Cord 2013; 51: 776-779.

13 Rabadi $\mathrm{MH}$, Aston C. Complications and urologic risks of neurogenic bladder in veterans with traumatic spinal cord injury. Spinal Cord 2015; 53: 200-203.

14 Singh R, Rohilla RK, Sangwan K, Siwach R, Magu NK, Sangwan SS. Bladder management methods and urological complications in spinal cord injury patients. Indian J Orthop 2011; 45: 141-147.

15 National Institute on Disability and Rehabilitation Research Consensus Validation Conference. The prevention and management of urinary tract infections among people with spinal cord injuries. J Am Paraplegia Soc 1992; 15: 194-204.

16 DeVivo MJ, Biering-Sorensen F, New P, Chen Y. Standardization of data analysis and reporting of results from the International Spinal Cord Injury Core Data Set. Spinal Cord 2011; 49: 596-599.

17 Mitsui T, Minami K, Furuno T, Morita H, Koyanagi T. Is suprapubic cystostomy an optimal urinary management in high quadriplegics? A comparative study of suprapubic cystostomy and clean intermittent catheterization. Eur Urol 2000; 38: 434-438.

18 Wefer B, Ehlken B, Bremer J, Burgdorfer H, Domurath B, Hampel C et al. Treatment outcomes and resource use of patients with neurogenic detrusor overactivity receiving botulinum toxin A (BOTOX) therapy in Germany. World J Urol 2010; 28: 385-390.

19 Game X, Castel-Lacanal E, Bentaleb Y, Thiry-Escudie I, De Boissezon X, Malavaud B et al. Botulinum toxin A detrusor injections in patients with neurogenic detrusor overactivity significantly decrease the incidence of symptomatic urinary tract infections. Eur Urol 2008; 53: 613-618.

20 Pannek J. Prophylaxis of urinary tract infections in subjects with spinal cord injury and bladder function disorders-current clinical practice. Aktuelle Urol 2012; 43: $55-58$.

21 Jepson RG, Williams G, Craig JC. Cranberries for preventing urinary tract infections. Cochrane Database Syst Rev 2012; 10: CD001321.

22 D'Hondt F, Everaert K. Urinary tract infections in patients with spinal cord injuries. Curr Infect Dis Rep 2011; 13: 544-551.

23 Niel-Weise BS, van den Broek PJ, da Silva EM, Silva LA. Urinary catheter policies for long-term bladder drainage. Cochrane Database Syst Rev 2012; 8: CD004201.

24 Morton SC, Shekelle PG, Adams JL, Bennett C, Dobkin BH, Montgomerie J et al. Antimicrobial prophylaxis for urinary tract infection in persons with spinal cord dysfunction. Arch Phys Med Rehabil 2002; 83: 129-138.

25 Massa LM, Hoffman JM, Cardenas DD. Validity, accuracy, and predictive value of urinary tract infection signs and symptoms in individuals with spinal cord injury on intermittent catheterization. J Spinal Cord Med 2009; 32: 568-573.

26 Bartel P, Krebs J, Wollner J, Gocking K, Pannek J. Bladder stones in patients with spinal cord injury: a long-term study. Spinal Cord 2014; 52: 295-297.

27 West DA, Cummings JM, Longo WE, Virgo KS, Johnson FE, Parra RO. Role of chronic catheterization in the development of bladder cancer in patients with spinal cord injury. Urology 1999; 53: 292-297.

28 Stonehill WH, Dmochowski RR, Patterson AL, Cox CE. Risk factors for bladder tumors in spinal cord injury patients. J Urol 1996; 155: 1248-1250.

29 Stohrer M, Blok B, Castro-Diaz D, Chartier-Kastler E, Del Popolo G, Kramer G et al. EAU guidelines on neurogenic lower urinary tract dysfunction. Eur Urol 2009; 56: 81-88. 